\title{
Shrapnel superior vena cava injury: Case report
}

\author{
Abdulsalam Y. Taha \\ Department of Thoracic and Cardiovascular Surgery, School of Medicine and Sulaimania Teaching Hospital, Sulaimania, Region of \\ Kurdistan, Iraq; salamyt_1963@hotmail.com
}

Received 9 December 2013; revised 5 January 2014; accepted 3 February 2014

Copyright (c) 2014 Abdulsalam Y. Taha. This is an open access article distributed under the Creative Commons Attribution License, which permits unrestricted use, distribution, and reproduction in any medium, provided the original work is properly cited. In accordance of the Creative Commons Attribution License all Copyrights (C) 2014 are reserved for SCIRP and the owner of the intellectual property Abdulsalam Y. Taha. All Copyright (C) 2014 are guarded by law and by SCIRP as a guardian.

\section{ABSTRACT}

Shrapnel injuries of the superior vena cava (SVC) are extremely rare and highly lethal. The true incidence is not known since many patients succumb shortly after injury. The high fatality is due to difficulty in diagnosis, technical problems with repair and consequences of SVC clamping. Literature review revealed no shrapnel SVC injury reported before. This is a report of 30-year-old man from Kirkuk, Iraq, a victim of terroristic attack who was admitted to Sulaimania Teaching Hospital (STH) on June 2007 with shock, massive right-side haemothorax and a wound at base of neck following a shrapnel injury. Emergency right thoracotomy revealed clotted haemothorax and big shrapnel partly sealing a tear in SVC just above the entrance of the azygos vein. Severe bleeding ensued after extraction of the shrapnel. Attempts to control the hemorrhage and repair of the injury failed and unfortunately, the patient expired in the theatre. Though there is no pathognomonic sign of SVC injury, it should be suspected in patients presenting with shock and haemothorax. Successful repair is achievable with early diagnosis, aggressive resuscitation, early exploration, optimum operating conditions and special measures such as auto-transfusion and cardiopulmonary bypass.

\section{KEYWORDS}

\section{Superior Vena Cava; Shrapnel; Injury}

\section{INTRODUCTION}

Superior vena cava is vulnerable to injuries of different kinds. Most of the reported injuries are iatrogenic; resulting from placement of central venous catheters, insertion of pacemakers, stenting of SVC in SVC obstruction syndrome or placement of a filter in the SVC to prevent showering of emboli [1]. Blunt and penetrating trauma to SVC is rare and highly fatal [2]. Herein, we report on a patient with isolated SVC injury by big shrapnel who unfortunately expired in the operating theatre because of uncontrolled hemorrhage. The case is presented with review of up to date medical literature. The aim is to recognize methods of early detection and measures of successful surgical repair.

\section{CASE SUMMARY}

A 30-year-old man was transferred from Kirkuk to Sulaimania after a big terrorist explosion at June 2007. He had an injury by shrapnel to the right neck root. He arrived few hours after the explosion with right-sided tube thoracostomy draining about 1400 cc blood. On arrival, he was pale and mildly dyspnoeic. His blood pressure was low and pulse was rapid. Air entry was diminished on right chest. He had a wound $4 \mathrm{~cm}$ in size overlying the medial half of right clavicle. There were no other injuries. Chest radiograph revealed a moderate-sized clotted haemothorax and a big shell in upper chest. Lateral views were obtained twice but were of poor quality and thus did not reveal the shell. The patient was resuscitated and prepared for right thoracotomy to drain the clotted haemothorax, stop the source of bleeding and to deal with any intra-thoracic injuries.

General anesthesia was given via a single lumen endotracheal tube. Right thoracotomy was chosen as that was the side of bleeding. The chest was entered through 5th intercostals space. Large clots were found (about $1000 \mathrm{cc}$ ) in the pleural space posteriorly and removed completely. The lung was healthy. There was a big and bulky shell (3 $\mathrm{cm}$ in length) just above the junction of the azygos vein with the SVC with bleeding around it (Figure 1).

Once the shell was dislodged, severe bleeding started. 


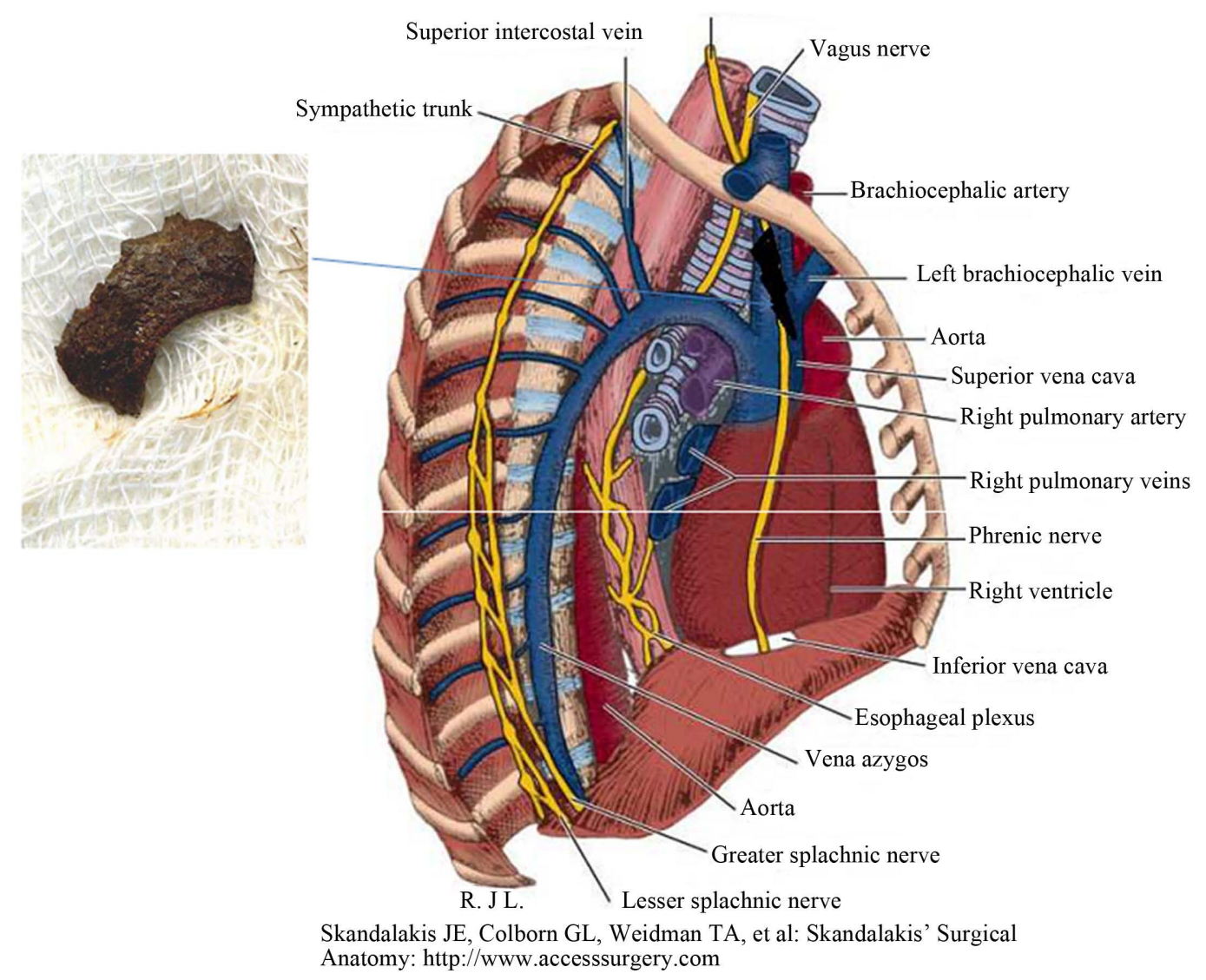

Figure 1. Shrapnel in the SVC. Inset: shrapnel.

The bleeding was initially controlled by manual compression while we prepared ourselves to repair the injury. This has failed; once the hand was released, the field was flooded with blood despite suction. A Foleys catheter is used to tampon the bleeding temporarily. The balloon could not be advanced distally enough because the tear was just at the confluence of innominate veins with the SVC. Repair was not possible with this big balloon in the tear. Surgical dissection was done and the SVC proximal to the tear was isolated and clamped but it was not possible to do so distally. Attempts to control the injury with a side clamp also failed. Meanwhile, the haemodynamic state of the patient was deteriorating. Ultimately, the patient expired.

\section{DISCUSSION}

Superior vena cava is the major vein draining the head, neck, upper thorax and upper limbs. It is a thin-walled vessel located in the superior part of the posterior mediastinum vulnerable to iatrogenic, blunt and penetrating injuries.

\subsection{Incidence}

Iatrogenic injuries are common, can be diagnosed preoperatively and the proper surgical approach chosen accordingly. In contrast, penetrating SVC injuries are rare but highly lethal. They are rarer following blunt chest trauma such as crushing and deceleration injuries [1]. The true incidence of penetrating wounds of the SVC as well as other great vessels is not known since many of these patients succumb shortly after injury and autopsy examination is not done in all patients dying after trauma [3]. Little has been written about cervicomediastinal venous injury [4]. We searched the English medical literature on the net; we obtained sporadic case reports of penetrating SVC injuries caused by stab wounds or gunshots but no case of SVC shrapnel injury was found.

In the study of Nair et al., 49 patients had cervico-mediastinal venous injuries. Eight patients (26.7\%) died. All of them were shocked on admission. Four of the 9 patients who were admitted in profound shock died on the operating table from exsanguinating haemorrhage. In the same study, there were 3 patients with SVC injuries. Two patients with stab wounds of SVC died on the operating table (66\% mortality) whilst the third patient survived an extensive laceration of SVC at the confluence of the brachiocephalic veins. He was subjected to venorrhaphy, narrowing the lumen of the SVC to $25 \%$ of 
its normal caliber. Postoperatively, he developed massive oedema of the arms, head and neck [4].

Symbas et al. reported on 36 patients with penetrating wounds of the great vessels treated at Grady Memorial Hospital during a 7-year period (1965-1972). One patient had 2 stab wounds of the SVC. Tangential partial occlusion of the SVC was used [3].

\subsection{Mortality}

SVC injuries, resulting from blunt or penetrating trauma will result in death before admission to the hospital in $45 \%$ of cases [1]. One third to one half of the remaining patients will die despite aggressive resuscitation and early surgical intervention [1]. The high mortality is due to difficulty in diagnosis and technical problems with repair [1]. Despite optimal care, thoracic venous injuries have a high mortality [1]. Exsanguinations may result without aggressive resuscitation and rapid surgical intervention.

\subsection{Diagnosis}

SVC injuries should be considered in any patient with a massive haemothorax. Unfortunately, there are no pathognomonic signs of azygos or SVC injuries [1]. Pulmonary, hilar and intercostal vessel injuries present with haemothorax as well. Subclavian, brachiocephalic and aortic injuries must also be considered [1]. Thoracic venous injuries usually present with signs of shock [1]. The blood pressure in these vessels is normally below systemic pressures, but the flow is high; therefore bleeding is usually massive [1]. Depending upon its exact site; SVC injury may result in bleeding into the pleural cavity, mediastinum or into the pericardium producing pericardial tamponade (when the entrance of SVC into the pericardium is injured) [1].

\subsection{Management}

Aggressive fluid resuscitation and blood transfusion are important to prevent haemodynamic collapse prior to transporting these patients to operating room where better lighting and equipment are available [1]. Auto-transfusion is helpful when blood loss is massive as it uses blood drained from the pleural cavity to restore circulating blood volume [1].

\subsection{Surgical Approach}

The approach is usually dictated by the clinical presentation [5]. A massive haemothorax mandates a right thoracotomy [5] as in our case. However, there is no incision which will satisfy all needs in emergency exploration of SVC injuries [3]. The trap door incision (antero-lateral thoracotomy, upper mid-sternotomy and lower neck incision) has the advantage that it can be extended to gain access to almost all great vessel wounds but is associated with greater morbidity to the patient and is more time-consuming for the surgeon [3]. Wide prepping and draping of the thorax and neck so that thoracotomy incision can be extended if needed, good exposure, adequate assistance, effective suction, sufficient blood for transfusion (or the use of intra-operative auto-transfusion) is essential for successful repair of great vessel injury [3].

\subsection{Clamping of SVC}

Partial caval clamping or clamping a chronically obstructed SVC is generally well tolerated; on the other hand, occlusion of a patent SVC may produce intracranial bleeding, brain oedema and damage, and a potentially lethal reduction of cardiac output [5].

\subsection{Repair vs. Ligation}

Repair should be undertaken in stable patients [4] whereas in haemodynamic unstable patient or when complex repair is needed, ligation is the preferred option [4]. However, ligation of the SVC is not the best management option particularly if there were no collateral veins to provide significant drainage of the upper body [5]. Successful ligation of the juxta-atrial portion of the SVC has been reported and attributed to the collateral effect of superior intercostals, azygos and hemiazygos veins [5].

\subsection{Special Measures}

Auto-transfusion greatly contributed to successful outcome in patients with massive intra-thoracic bleeding by promptly procuring sufficient quantities of blood [3]. This procedure has been proved to be safe and frequently life-saving for such patients [3].

The majority of caval injuries can be repaired without the need of cardiopulmonary bypass (CPB). However, median sternotomy and CPB may be required for injuries close to the heart where distal control is not tolerated and can be helpful in decompressing the venous system and allowing improved exposure [5].

Our patient unfortunately died despite surgical exploration and different measures used to control the haemorrhage such as balloon catheter and clamping of the SVC. Ligation was not used as it would not control back flow. Probably he could be saved if facilities of auto-transfusion and CPB were available.

\section{CONCLUSION}

Shrapnel SVC injury is extremely rare and highly fatal with no pathognomonic presentation. It should be sus- 
pected whenever massive haemothorax is encountered. Successful repair may be achieved with good operating conditions, proper lighting, effective suction, adequate assistance, good anaesthetic management and special measures such as auto-transfusion and CPB.

\section{CONFLICT OF INTEREST}

None.

\section{REFERENCES}

[1] Walsh, A. and Snyder, H.S. (1992) Azygos vein laceration following a vertical deceleration injury. The Journal of Emergency Medicine, 10, 35-37. http://dx.doi.org/10.1016/0736-4679(92)90008-H
[2] Ochsner, J.L., Crawford, E.S. and Debakey, M.E. (1961) Injuries to the vena cava caused by external trauma. Surgery, 9, 397-405.

[3] Symbas, P.N., Kaourias, E., Tyras, D.H. and Hatcher, C.R. (1974) Penetrating wounds of great vessels. Annals of Surgery, 179, 757-761.

http://dx.doi.org/10.1097/00000658-197405000-00031

[4] Nair, R., Robs, J.V. and Muckart, D.J. (2000) Management of penetrating cervicomedistinal venous trauma. European Journal of Vascular \& Endovascular Surgery, 19, 65-69. http://dx.doi.org/10.1053/ejvs.1999.0965

[5] Ekim, H. (2009) Management of penetrating superior caval vein injury: Case report. Pakistan Journal of Medical Sciences, 25, 869-871. 